EPJ Web of Conferences 32, 03009 (2012)

DOI: $10.1051 /$ epjconf/20123203009

(C) Owned by the authors, published by EDP Sciences, 2012

\title{
Survey of the TS-ECE Discrepancy and recent investigations in ICRF heated plasmas at Alcator C-Mod
}

\author{
A. E. White ${ }^{1}$, A. E. Hubbard ${ }^{1}$, J. W. Hughes ${ }^{1}$, P. T. Bonoli ${ }^{1}$, M. E. Austin ${ }^{2}$, A. Bader $^{3}$, R. W. \\ Harvey $^{4}$, Y. Lin ${ }^{1}$, Y. Ma ${ }^{1}$, M. L. Reinke ${ }^{5}$, S. M. Wolfe ${ }^{1}$, S. J. Wukitch ${ }^{1}$, and the Alcator C-Mod \\ team $^{1}$ \\ ${ }^{1}$ Massachusetts Institute of Technology, Cambridge, MA, USA \\ ${ }^{2}$ University of Texas, Austin, TX, USA \\ ${ }^{3}$ University of Wisconsin, Madison, WI, USA \\ ${ }^{4}$ CompX, CA, USA \\ ${ }^{5}$ Oak Ridge Institute for Science and Education (ORISE), Oak Ridge, TN, USA
}

\begin{abstract}
This paper reports on a new investigation of the long-standing, unresolved discrepancy between Thomson Scattering (TS) and Electron Cyclotron Emission (ECE) measurements of electron temperature in high temperature tokamak plasmas. At the Alcator C-Mod tokamak, ion cyclotron range of frequency (ICRF) heating is used to produce high temperature conditions where the TS- ECE discrepancy, as observed in the past at JET and TFTR, should appear. Plasmas with $\mathrm{T}_{\mathrm{e}}(0)$ up to $8 \mathrm{keV}$ are obtained using three different heating scenarios: Ion Cyclotron Resonance Heating (ICRH), ICRF mode conversion heating and a combination of the two heating methods. This is done in order to explore the hypothesis that ICRH-generated fast ions may be related to the discrepancy. In all high temperature cases at C-Mod, we find no evidence for the type of discrepancy reported at JET and TFTR. Here we present the C-Mod results along with a summary of past work on the TS-ECE discrepancy.
\end{abstract}

\section{Introduction}

In some tokamak experiments featuring strong neutral beam and ion cyclotron range of frequency (ICRF) auxiliary heating have shown the existence of a clear discrepancy between Te measured by TS and ECE when Te $>7 \mathrm{keVinTFTR} \mathrm{[1,2]} \mathrm{and} \mathrm{when} \mathrm{Te}>5 \mathrm{keV}$ in JET[3,4]. There is presently no explanation for the TS-ECE discrepancy that is observed at either JET or TFTR, but it does not appear to be caused by instrumental effects or measurement errors [3]. Usually, temperature measurements made with Thomson Scattering (TS) and Electron Cyclotron Emission (ECE) diagnostics are typically in excellent agreement when $\mathrm{Te}<5 \mathrm{keV}$. The existence of a temperaturedependent TS-ECE discrepancy presents a significant challenge for ITER where core electron temperatures above $20 \mathrm{keV}$ are expected. The discrepancy is one strong motivation for the consideration of an oblique ECE instrument for ITER [5,6]. Understanding the discrepancy has been called the next major advance for ECE [7]. In this paper, we present first a summary of past studies of the TS-ECE discrepancy. Then we describe the experiments performed at C-Mod to probe the discrepancy. We mention some possible paths for future work on this topic.

This is an Open Access article distributed under the terms of the Creative Commons Attribution License 2.0, which permits unrestricted use, distribution, and reproduction in any medium, provided the original work is properly cited. 


\section{Survey of work on the TS-ECE discrepancy}

The discrepancy that was observed at TFTR and JET is characterized by ECE measurements of electron temperature that are systematically higher than the TS measurements at high temperatures. The discrepancy increases linearly with increasing temperature, but there is good agreement between the two measurements at low temperatures [3, 6, 8]. Differences between TS and ECE measurements are often observed in tokamak plasmas with strong Electron Cyclotron Resonance Heating (ECRH), Electron Cyclotron Current Drive (ECCD) and Lower Hybrid Current Drive (LHCD). In these cases, the presence of non-thermal electrons can enhance the EC emission above thermal levels. Such differences are reasonably well understood $[9,10]$, in contrast to the JET and TFTR "discrepancy".

The TS-ECE discrepancy was first reported for supershot D-T plasmas at TFTR [1], wih combined NBI and ICRF heating. The discrepancy appeared as a systematic difference, with ECE measuring temperatures above TS, but only when $\mathrm{T}_{e}(0)>7 \mathrm{keV}$. No cause could be identified [2]. The TS-ECE discrepancy has also been observed with NBI-only in TFTR [6]. NBI injection produces fast ions, which over a certain range of parameters transfer energy predominantly to electrons. Because of the potential strong coupling between the fast ions and the bulk electrons, was suggested a distortion of the electron distribution function could be produced at low energies, leading to the observed TS-ECE discrepancy $[1,7]$. However, there is no proposed mechanism for how this would be produced or maintained against collisional relaxation.

Later, the discrepancy was identified at JET in plasmas with combined NBI and ICRH, and extensive work was done to show that it was not caused by instrumental uncertainties, and that it was indeed a real effect [3]. One key aspect of the JET studies are theoretical calculations showing that a distorted electron distribution function could reproduce both the ECE measurements and the TS measurements [11]. On JET, studies with full ECE spectral measurements from a Michelson interferometer showed that temperature measured from the 2 nd harmonic was not in agreement with the 3rd harmonic when a Maxwellian distribution function was assumed. But when a model electron distribution was introduced, with a flattening at low-energies (a non- Maxwellian bulk), the effective temperature measured from the modeled 2nd and 3rd harmonics was in agreement, and also, the TS and ECE measurements would also agree $[3,11]$. However, no physical mechanism has been proposed to explain how a distortion of the electron distribution function near thermal velocities is sustained.

Despite the existence of a real (not due to instrumental error or uncertainty) discrepancy at JET and TFTR, is fair to say that the discrepancy may not be a universal feature of high temperature plasmas. This makes predicting its existence in ITER plasmas problematic. Further complicating the issue, is that the discrepancy at JET was observed in hybrid scenarios featuring combined NBI and ICRH in the years 2001 and 2004 but the discrepancy was not reproduced in experiments in the year 2006 featuring similar plasma conditions $[4,8]$. One difference between these two sets of discharges identified by the JET team is the hydrogen minority concentration. In the plasmas where the discrepancy was observed, the hydrogen concentration was low, $3 \%$, and when the discrepancy was not observed, the hydrogen fraction was higher, 8\% [8]. The effective tail temperature of ICRHgenerated fast ions is very sensitive to the minority fraction, and for low hydrogen fractions on JET, the tail temperature can be very high, exceeding Teff $=500 \mathrm{keV}[8,12,13]$. The observation that the JET TS-ECE discrepancy is correlated with very high-energy ion tails in the plasmas, suggested that the fast ions play a role in causing the discrepancy $[3,4,8]$.

Further work has gone into diagnosing the presence of the non-Maxwellian bulk at JET. For example, recently a new oblique ECE system was deployed at JET [14]. The authors examined a variety of high temperature plasmas with fast-ion populations (NBI-only and NBI+ICRH, with $\mathrm{Te}(0)$ $<7 \mathrm{keV}$ ) and found no evidence of deviation from Maxwellian behavior in the data, using both the radial and oblique views. [14]. 
Finally, the most recent development for the JET TS-ECE discrepancy studies is the recent on-line publication (on arXiv.org) of a paper describing large-scale drifts is electron temperature measurements on JET plasmas [15]. The drifts are reported to cause overall uncertainties in the central temperature measurement of between $16-22 \%$, and the paper finds that difference between TS-ECE measurements varies with time, including seasonal variations [15]. Given this development, it is very important that current tokamak experiments explore the temperature dependent discrepancy reported at TFTR and JET: first to verify the general existence conditions, and then ideally, to identify the cause of the discrepancy and correct for it.

\section{Experimental Results from Alcator C-Mod}

Three separate ECE instruments are used at Alcator C-Mod: a 9-channel ECE grating polychromator (GPC-1) [16], a 19-channel ECE grating polychromator (GPC-2) [17, 18], and an ECE Michelson interferometer [19]. The GPC instruments are tuned to measure 2nd harmonic X-mode EC emission.
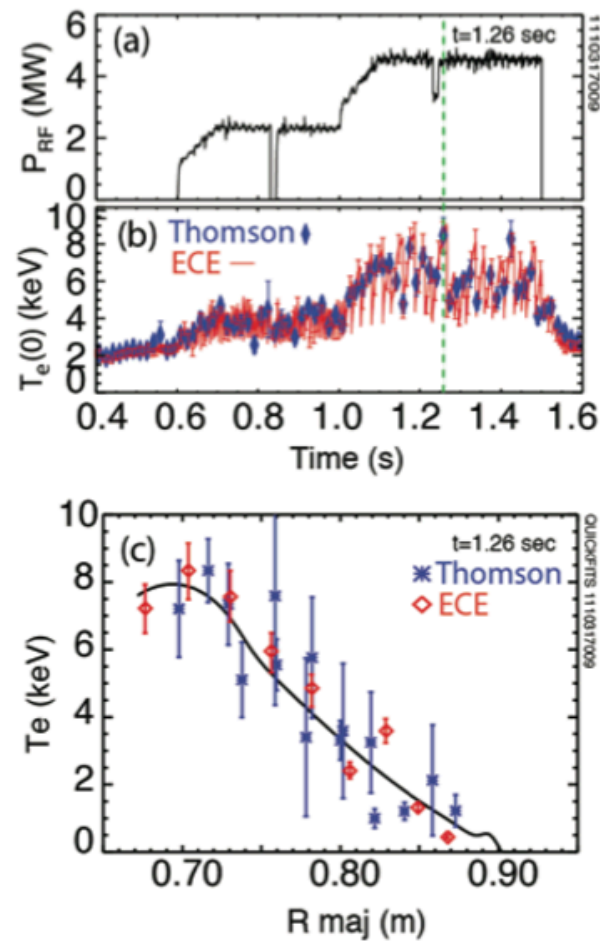

Fig. 1. (a) Time trace of RF power, (b) central TS (blue-diamond) and ECE data from GPC-1 (red-line) as a function of time, and (c) the electron temperature profile at $\mathrm{t}=1.26 \mathrm{~s}$ for a high temperature plasma featuring combined mode conversion and minority heating.
The Michelson interferometer is absolutely calibrated. The grating polychromators are crosscalibrated to the Michelson over a wide frequency range. All ECE systems share the same optics and view the plasma perpendicularly at the midplane from the low field side; none of the views are oblique. The Thomson Scattering (TS) diagnostic used in this experiment [20] has lasers directed vertically into the plasma and spectra are measured from the outboard midplane. The TS polychromator has a filter arrangement that can reliably measure temperatures up to $\mathrm{Te}=20 \mathrm{keV}$ [20]. Analysis of the TS measured spectrum assumes that the electron distribution is thermal.

There are two primary ICRF heating configurations used at C-Mod: hydrogen minority ICRH (fast ions transfer energy to plasma via electron drag and collisions with thermal ions) and mode conversion heating (direct electron Landau damping of the mode converted fast wave). Details of the ICRF systems are reviewed in Ref. [21]. CMod has a Lower Hybrid (LH) system [21], but this is not used in these experiments.

Plasmas in the experiments are all sawtoothing L-mode and I-mode discharges [22], with $\left\langle\mathrm{n}_{\mathrm{e}}\right\rangle=0.9-1.5 \times 10^{20} \mathrm{~m}^{-3}, \mathrm{PRF}=2-5 \mathrm{MW}$, and Ip $=1.0-1.3$ MA. Three different RF configurations used in order to isolate the effects of fast ions on the TS-ECE discrepancy. In the first heating configuration, Mode Conversion ICRF heating is done by running two species plasmas $\mathrm{D}(3 \mathrm{He})$ at $\mathrm{Bt}$ $=7.8-8.0 \mathrm{~T}$, with two antennas set to $80.5 \mathrm{MHz}$ and $80.0 \mathrm{MHz}$; and a third antenna set to $78.0 \mathrm{MHz}$. High ${ }^{3} \mathrm{He}$ minority fractions (10-15\%, scanned shot by shot) are used so that a large percentage of the wave power is mode converted and only a small percentage is absorbed by the minority ions. In this configuration, the mode conversion layer is close to the magnetic axis. In the second configuration, a combination of mode conversion and minority heating can be accomplished by running three species plasmas $\mathrm{D}\left(\mathrm{H},{ }^{3} \mathrm{He}\right)$ at $\mathrm{Bt}=5.0-5.8$ T. Here two antennas are set to $80.5 \mathrm{MHz}$ and $80.0 \mathrm{MHz}$, with the third set to $50 \mathrm{MHz}$. In this 
configuration, the mode conversion layer is close to the magnetic axis and the Hydrogen resonance layer is off-axis, on the high field side. In these cases the Hydrogen minority fraction is $\approx 4-6 \%$ and the Helium fraction is $>15 \%$. In the third configuration, minority heating is done in a two species plasmas $\mathrm{D}(\mathrm{H})$ at $\mathrm{Bt}=5.4-5.8 \mathrm{~T}$. In this case, two antennas are again set to $80.5 \mathrm{MHz}$ and $80.0 \mathrm{MHz}$, with the third antenna set to $78.0 \mathrm{MHz}$. At 5.8 T, the Hydrogen resonance layer is offaxis, on the low field side. The Hydrogen minority fraction is $\approx 4-6 \%$. For all the ICRH plasmas considered here, the critical energy is Wcrit $\approx 13.5 \mathrm{~T}_{\mathrm{e}}$ so that fast ions generated in the minority heating configuration slow down predominantly due to electron drag.

Shown in Figure 1 is data from a high temperature plasma featuring both RF mode conversion and minority heating: (a) time trace of RF power, (b) central TS and ECE data (from GPC-1) as a function of time, and (c) the electron temperature profile at $t=1.26$ s. Figure 1 (b)
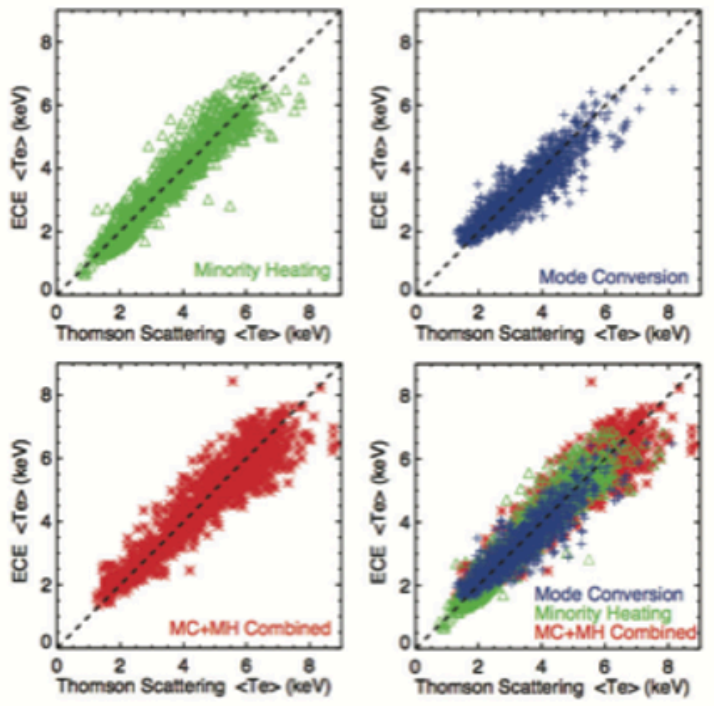

Fig. 2. Central temperature measured with ECE (using both GPC1 and GPC2 data) compared with Thomson Scattering. All pairs of ECE and TS data from within the radial range $0.68 \mathrm{~m}<\mathrm{Rmaj}<0.72 \mathrm{~m}$ are plotted. The paired measurements are made within $1 \mathrm{~ms}$ of each other, and are separated in radius by less than $1 \mathrm{~cm}$ shows that there is excellent agreement between the central TS and ECE (GPC-1) measurements at all times in the discharge: during the Ohmic phase before RF is turned on, $\mathrm{t}<0.6 \mathrm{sec}$, with $2.5 \mathrm{MW} \mathrm{RF}$ power (mode conversion heating) at low temperatures, $\operatorname{Te}(0)<5 \mathrm{keV}$, between 0.6 $<\mathrm{t}<1.0 \mathrm{sec}$, and with $4.5 \mathrm{MW}$ RF power (combined mode conversion and minority heating) at high temperatures, $\operatorname{Te}(0)>5$ $\mathrm{keV}$, between $1.0<\mathrm{t}<1.5 \mathrm{sec}$.

To compare the TS and ECE data points one-to-one, we use data from both GPC systems. The TS data sets the time base for the comparisons, since the TS data are acquired every $16 \mathrm{~ms}$ and GPC data are acquired every $50 \mu \mathrm{s}$. The ECE data are averaged over $1 \mathrm{~ms}$ at the time of comparison. As can be seen in Figure 1 (b), the ECE data (red) track the sawteeth throughout the shot, but due to the slower TS sampling rate the TS data (blue) measure the highest temperature (peak of a sawtooth) less frequently. This limits the number of data points obtained at high temperature that can be used for a one-to-one comparison in any single discharge. The large sawteeth crashes occurring during interferograms can lead to spurious structure in the measured profiles. For these reasons, the slowly scanning Michelson interferometer data were not used to investigate the discrepancy: there were too few spectra measured at the high temperature times to form a complete data set. At low temperatures, there is excellent agreement between the TS, GPC, and 2nd harmonic Michelson profiles.

Figure 1 (c) shows the electron temperature profile data plotted versus major radius. The Thomson data (blue) and ECE data (red) are in good agreement. The black solid line is a fit to the data shown and the edge TS data (not shown). Experimental error bars are shown. The TS data are mapped from real space into flux coordinates and are then compared with the GPC data, which are also mapped from real space into flux coordinates using the EFIT reconstruction [23]. Errors in radial mapping near the magnetic axis due to uncertainties in the equilibrium reconstruction are estimated to be between $1.0 \mathrm{~cm}$ and $1.5 \mathrm{~cm}$. The ECE data are mapped using the cold resonance location, which means that due to relativistic broadening, the ECE sample volume extends $1.0-1.5$ 
$\mathrm{cm}$ in the radial direction on the high field side of the data points shown in the temperature profile. The ECE sample volume near the magnetic axis has a vertical extent of roughly $4 \mathrm{~cm}$ due to the GPC antenna pattern. The spatial resolution of the TS diagnostic is subcentimeter: a few $\mathrm{mm}$ in the radial direction with a vertical extent of the scattering volume $\sim 6 \mathrm{~mm}$ at the center of the plasma. All the discharges in this study are relatively low-density discharges for C-Mod $\left(\langle\mathrm{ne}\rangle<1.5 \times 10^{20} \mathrm{~m}^{-3}\right)$, and error bars on TS are atypically large (Figure 1) because of the reduced signal to noise.

The TS-ECE comparison results from all three heating configurations are summarized in Figure 2. Central temperature measured with ECE (using both GPC1 and GPC2 data) is plotted on the vertical axis and central temperature measured with Thomson Scattering is plotted on the horizontal axis. All pairs of ECE and TS data from within the radial range $0.68 \mathrm{~m}<\mathrm{Rmaj}<0.72 \mathrm{~m}$ near the magnetic axis are plotted. We restrict the comparison to pairs of TS and ECE measurements made within $1 \mathrm{~ms}$ of each other, and separated in radius by less than $1 \mathrm{~cm}$. The black-dashed line indicates the unity line, not a regression line or fit to the data.

Plotting the data in the format of Figure 3 shows qualitatively that there appears to be no gross systematic difference between the two measurements over the temperature range $1.5<\mathrm{Te}<$ $8.0 \mathrm{keV}$. From this figure, there is already evidence that the type of TS-ECE discrepancy seen at JET and TFTR is not present in the C-Mod data. For the TS-ECE discrepancy on JET and TFTR, the TS and ECE data would begin to disagree outside error bars only at higher temperature, while agreeing within error bars at lower temperature. A statistical model can used to compare measurements from each instrument quantitatively to determine if any evidence for the TS-ECE discrepancy exists [24, $25,26]$. The application of this statistical model to the C-Mod data is described in detail in Ref. [27].

\section{Conclusions and future work}

At Alcator C-Mod, we have found no evidence of the type of TS-ECE discrepancy observed at JET and TFTR, with or without the presence of fast ions, up to Te(0) $<8 \mathrm{keV}$. The TS and ECE measurements of electron temperature are found to agree to within experimental uncertainties at low and high temperatures. Disagreements between TS and ECE measurements that are observed can be attributed to random errors and systematic errors, e.g. in the calibration of one or both instruments. Future modeling work using Fokker-Planck codes such as CQL3D [33] to self-consistently evolve the electron distribution function and the fast ion distribution function simultaneously in order to probe the physics of how fast ions affect the electron distribution function could help shed more light on the possible effects of fast ions. Future experiments to investigate the discrepancy should include, when possible, measurements of, or constraints on, the electron temperature using other diagnostics (e.g. exploiting relativistic effects to extract Te from reflectometry [28] or perhaps using an x-ray crystal spectrometer to assess Te from impurity lines [29].

\section{References}

[1] G. Taylor and et al. Electron cyclotron emission measurements on high beta TFTR plasmas. Proc. 9th Joint Workshop on ECE and ECRH (Borrego Springs, USA, 1995) ed. J. LOHR (World Scientific, Singapore, 1995), page $485,1995$.

[2] I. Fidone, G. Giruzzi, and G. Taylor. Plasma diagnostics in the Tokamak Fusion Test Reactor using emission of electron cyclotron radiation at arbitrary frequencies. Physics of Plasmas, 3:2331, 1996.

[3] E. de la Luna, V. Krivenski, G. Giruzzi, C. Gowers, R. Prentice, J. M. Travere, and M. Zerbini. Impact of bulk non-maxwellian electrons on electron temperature measurements (invited). Rev. Sci. Instrum., 74:14141420, 2003.

[4] K. V. Beausang, S. L. Prunty, R. Scannell, M. N. Beurskens, M. J. Walsh, E. de la Luna, and JET EFDA Contributors. Detecting non-maxwellian electron velocity distributions at JET by high resolution Thomson scattering. Rev. Sci. Instrum., 82:033514, 2011.

[5] E. de la Luna and JET-EFDA contributors. Physics of ECE temperature measurements and prospects for ITER. volume 988, pages 62-72. AIP Conference Proceedings, 2007.

[6] G. Taylor and R. W. Harvey. Assessment of an oblique ECE diagnostic for ITER. Fusion Science and 
Technology, 55:64, 2009.

[7] A. Costley. ECE: The story so far. Proc. 15th Joint Workshop on ECE and ECRH (Yosemite, USA, 2008) ed. J. LOHR (World Scientific, Singapore, 2008), page 3, 2008.

[8] E. de la Luna and et al. Recent results on the discrepancy between electron temperature measurements in high temperature plasmas in JET. Proc. 15th Joint Workshop on ECE and ECRH (Yosemite, USA, 2008) ed. J. LOHR (World Scientific, Singapore, 2008), page 123, 2008.

[9] V. Krivenski. Electron cyclotron emission by non-maxwellian bulk distribution functions. Fusion Engineering and Design, 53:23, 2001.

[10] S Coda, I Klimanov, S Alberti, G Arnoux, P Blanchard, A Fasoli, and the TCV team. The effect of ECRH on the electron velocity distribution function. Plasma Physics and Controlled Fusion, 48(12B):B359, 2006.

[11] V. Krivenski, E. de la Luna, and G. Giruzzi1. Evidence of non-maxwellian electron bulk distributions on JET. 29th EPS Conference on Plasma Phys. and Contr. Fusion Montreux, 17-21 June 2002, ECA, 26B(O):1.03, 2002.

[12] V. G. Kiptily and et al. $\gamma$-ray diagnostics of energetic ions in JET. Nuclear Fusion, 42:999, 2002

[13] V. G. Kiptily and et al. Recent progress in fast ion studies on JET. Nuclear Fusion, 49:065030, 2009

[14] L. Figini, S. Garavaglia, E. De La Luna, D. Farina, P. Platania, A. Simonetto, C. Sozzi, and JET-EFDA Contributors. Measure of electron cyclotron emission at multiple angles in high Te plasmas of JET. Review of Scientific Instruments, 81(10):10D937, 2010

[15] Thomas Gerbaud, et al. Large-scale drifts observed on electron temperature measurements on JET plasmasarXiv:1205.2969v1 [physics.plasm-ph] 14 May 2012

[16] [P. J. O'Shea, A. E. Hubbard, and the Alcator C-Mod Group. Nine channel polychromator for fast te measurements on Alcator C-Mod. Proc. 9th Joint Workshop on ECE and ECRH (Borrego

Springs, USA, 1995) ed. J. LOHR (World Scientific, Singapore, 1995), page 7, 1995.

[17] A. Janos and et al. Operation of two grating polychromators on TFTR and new observations of magnetohydrodynamic phenomena. Rev. Sci. Instrum, 66:668, 1995.

[18] G. Taylor and et al. Electron power deposition measurements during ion cyclotron range of frequency heating on C-Mod. AIP Conf. Proc., 485:490, 1999.

[19] S. E. Kissel T. C. hsu and I. H. Hutchinson. A novel fast scan Michelson interferometer for ECE diagnostic applications on Alcator C-mod. Proceedings of the 7th Joint Workshop and IAEA Technical Committee on Electron Cyclotron Emission and Electron Cyclotron Resonance Heating, page 31, 1990.

[20] J. W. Hughes, D. Mossessian, K. Zhurovich, M. DeMaria, K. Jensen, and A. Hubbard. Thomson scattering upgrades on Alcator C-Mod. Review of Scientific Instruments, 74(3):1667-1670, 2003.

[21] P. T. Bonoli and et al. Wave-particle studies in the ion cyclotron and lower hybrid ranges of frequencies in Alcator C-Mod. Fusion Science and Technology, 51:401, 2007.

[22] D.G. Whyte, A.E. Hubbard, J.W. Hughes, B. Lipschultz, J.E. Rice, E.S. Marmar, M. Greenwald, I. Cziegler, A. Dominguez, T. Golfinopoulos, N. Howard, L. Lin, R.M. McDermott, M. Porkolab, M.L. Reinke, J. Terry, N.Tsujii, S. Wolfe, S. Wukitch, Y. Lin, and the Alcator C-Mod Team. I- mode: an H-mode energy confinement regime with L-mode particle transport in Alcator C-Mod. Nuclear Fusion, 50(10):105005, 2010.

[23] L.L. Lao, H. St. John, R.D. Stambaugh, A.G. Kellman, and W. Pfeiffer. Reconstruction of current profile parameters and plasma shapes in tokamaks. Nucl. Fusion, 25(11):1611, 1985.

[24] D. G. Altman and J. M. Bland. Measurement in medicine: The analysis of method comparison studies. Journal of the Royal Statistical Society. Series D (The Statistician), 32(3):307, 1983.

[25] D. G. Altman and J. M. Bland. Statistical methods for assessing agreement between two methods of clinical measurement. Lancet, i:307, 1986.

[26] A. Kinsella. Estimating method precisions. Journal of the Royal Statistical Society. Series D

(The Statistician), 35(4):421, 1986.

[27] A. E. White, et al., Investigation of the TS-ECE Discrepancy in ICRF heated plasmas at Alcator C-Mod, Accepted for publication, Nuclear Fusion (2012)

[28] L. Zeng, W. A. Peebles, E. J. Doyle, T. L. Rhodes, and G. Wang. Relativistic effects on reconstruction of density profiles via reflectometry in ITER and potential for electron temperature measurements. Plasma Phys. Control. Fusion, 49:12771287, 2007.

[29] N. Pablant and et al. First results from the high-resolution x-ray imaging crystal spectrometer on the Large Helical Device. In 53rd Annual Meeting of the APS Division of Plasma Physics,volume 56, 2011. 